\title{
Development and modification of new fascial plane blocks
}

\author{
Kunihisa Hotta ${ }^{1}$ \\ Received: 5 October 2021 / Accepted: 5 October 2021 / Published online: 15 November 2021 \\ (c) The Author(s) under exclusive licence to Japanese Society of Anesthesiologists 2021
}

Keywords Fascial plane block · Ultrasound · Quadratus lumborum block · Erector spinae plane block

In recent years, ultrasound-guided fascial plane blocks (FPBs) have become commonly used in the field of anesthesia and pain medicine, and the development and modification of new FPB techniques are happening rapidly. New FPB techniques developed based on innovative ideas may have some benefits, including superior efficacy and safety, which may ultimately change the standard of care. Such new approaches should be used with caution, however, because the differences in expected sensory block areas, as well as technical difficulties and complications, between new FPBs and conventional blocks have yet to be determined.

The use of ultrasound has the potential to broadly expand the use of FPB in pain management. Ultrasound provides visualization that the injectate is administered to the proper fascial plane and enhances the accuracy of the FPB procedure, which had previously been performed using the tactile sensation of fascial plane entry. As a result, conventional FPBs such as the fascia iliaca compartment block and the transversus abdominis plane (TAP) block have become more reliable with ultrasound guidance. Moreover, the advanced understanding of the sonoanatomy of fascial planes and surrounding structures may have contributed to the development of truncal FPBs that can be safer and easier than the traditional epidural block. The concept of multimodal analgesia, the need for alternatives to epidural anesthesia, and the popularity of minimally invasive surgery have also accelerated the development of ultrasound-guided FPBs. Ultrasound-guided FPBs, unlike selective peripheral nerve blocks, do not require direct imaging of the target nerves, instead ensuring sufficient dermatomal coverage of sensory blockade by injection of much larger volumes of LA

Kunihisa Hotta

hottak@jichi.ac.jp

1 Department of Anesthesiology and Critical Care Medicine, Jichi Medical University School of Medicine, 3311-1 Yakushiji, Shimotsuke, Tochigi 329-0498, Japan than is necessary for peripheral nerve blocks. In addition to the spread throughout the fascial plane to which the LA is administered, the spread of LA to adjacent tissues may also be a mechanism of analgesic action in some FPBs. The drawback with this approach is that it is nearly impossible to monitor the entire spread of LA and control it intentionally. Thus, the volume of LA injected may be larger than is necessary for adequate nerve blockade.

The quadratus lumborum (QL) block is a relatively new FPB and was developed based on the concept of the original TAP block, which uses the triangle of Petit as the main anatomical landmark [1]. In the QL block, the LA is administered to the thoracolumbar fascia (TLF) that surrounds the QL muscle. The QL block mainly covers the subcostal, ilioinguinal, and iliohypogastric nerves, which are on the ventral aspect of the QL muscle. One clinical study found that the analgesic effects of the QL block are superior to those of the ultrasound-guided lateral TAP block in patients undergoing Cesarean Section [2]. Use of magnetic resonance imaging has demonstrated that contrast media administered to the TLF at the lumbar level can extend into the thoracic paravertebral space (PVS) [3], as has a cadaver study [4]. The spread of LA into the thoracic PVS is presumed to be the mechanism of the extensive sensory blockade provided by the QL block.

Several approaches to the injection site in QL block have been reported: QLB1 (lateral approach), QLB2 (posterior approach), QLB3 (anterior approach), and the intramuscular approach. The spread of LA differs between the different techniques, leading to variation in the sensory block area. Previous clinical trials have assessed the analgesic effect of the QL blocks in various types of surgery [5-9]. In the anterior approach, the LA is administered to the fascial plane between the QL and psoas major muscles and can block the lumbar plexus [10]. In a study comparing the QLB2 and intramuscular approach, the spread of injectate into the thoracic PVS was less extensive in the intramuscular approach 
than it was in the QLB2 [3]. The spread of LA to the TLF seems important for the broader spread to the thoracic PVS, and some studies have failed to demonstrate the spread of injectate into the thoracic PVS [11, 12]. Thus, it is reasonable to assume that large amounts of injectate do not always reach the thoracic PVS with these QL block approaches.

The fascial plane posterior to the lateral and medial arcuate ligaments has been proposed as a route for LA to reach the thoracic PVS in the QL block [4]. To enhance analgesic effects at the thoracic nerve level, more LA is expected to reach the thoracic PVS when administered near the lateral arcuate ligament. The subcostal approach to the QL block reported by Elsharkawy et al. has shown extensive sensory block between T6-7 and L1-2 by moving the injection site more cephalad, to just below the 12th rib [13]. The parasagittal approach at the lateral supra-arcuate ligament reported by Shi et al. was a modified anterior approach aimed at directing the injectate into the lower thoracic PVS [14]. There is not yet enough data on the safety and efficacy of these cephalad approaches. Future studies are needed to assess whether there are differences in sensory block areas and the amount of LA required between the conventional QL block approaches and the new cephalad approaches.

The erector spinae plane (ESP) block is also a newly developed ultrasound-guided FPB [15, 16]. As in the QL block, the spread of LA beyond the fascial plane to adjacent tissues has been observed in the ESP block. In the thoracic ESP block, LA is administered to the fascial plane between the erector spinae muscle and the transverse process of the vertebrae, and most of the LA remains in the posterior vertebral region, blocking the dorsal rami of the thoracic nerves. It has been noted that some LA moves to the anterior region, reaches the thoracic PVS, and blocks the ventral rami of the thoracic nerves [17]. The ESP block can be performed at either the cervical, thoracic, or lumbar levels. The cervical ESP block has been reported to cause the spread of LA to the prevertebral compartment and can be used as a perioperative analgesic method in patients undergoing shoulder surgery [18]. The costotransverse foramen block has been reported as an alternative to thoracic paravertebral block. In this block, more injectate is expected to spread anteriorly into the thoracic PVS than is seen with the thoracic ESP block [19].

Many ultrasound-guided FPBs have been developed over the last two decades and are now widely used. Evaluation of the safety and efficacy of the new FPB techniques scientifically and systematically will be necessary to guide clinical decisions about the use of these blocks. Agreement among practitioners on the nomenclature for these new FPBs will also be required. In some instances, similar FPB techniques may have equivalent efficacy and safety, so much so that it may not be necessary to make a strict distinction between them. In other FPBs, newly developed techniques may replace traditional ones. It is expected that the development and modification of FPBs will continue, and new FPB techniques based on innovative ideas will improve clinical outcomes.

\section{References}

1. Blanco R, Ansari T, Girgis E. Quadratus lumborum block for postoperative pain after caesarean section: a randomised controlled trial. Eur J Anaesthesiol. 2015;32:812-8.

2. Blanco R, Ansari T, Riad W, Shetty N. Quadratus lumborum block versus transversus abdominis plane block for postoperative pain after cesarean delivery: a randomized controlled trial. Reg Anesth Pain Med. 2016;41:757-62.

3. Tamura T, Yokota S, Ito S, Shibata Y, Nishiwaki K. Local anesthetic spread into the paravertebral space with two types of quadratus lumborum blocks: a crossover volunteer study. J Anesth. 2019;33:26-32.

4. Dam M, Moriggl B, Hansen CK, Hoermann R, Bendtsen TF, Børglum J. The pathway of injectate spread with the transmuscular qadratus lumborum block: a cadaver study. Anesth Analg. 2017;125:303-12.

5. Aoyama Y, Sakura S, Abe S, Tadenuma S, Saito Y. Continuous quadratus lumborum block and femoral nerve block for total hip arthroplasty: a randomized study. J Anesth. 2020;34:413-20.

6. Tan HS, Taylor C, Weikel D, Barton K, Habib AS. Quadratus lumborum block for postoperative analgesia after cesarean delivery: a systematic review with meta-analysis and trial-sequential analysis. J Clin Anesth. 2020;67:110003.

7. Little C, Rahman S. Quadratus lumborum blocks in nephrectomy: a narrative review. Local Reg Anesth. 2021;14:57-65.

8. Singh NP, Makkar JK, Borle A, Monks D, Goudra BG, ZorrillaVaca A, Singh PM. The analgesic efficacy of quadratus lumborum block in caesarean delivery: a meta-analysis and trial sequential analysis. J Anesth. 2020;34:814-24.

9. Kinjo S, Kolodzie K, Dong K, Zhang AL. The effects of transmuscular quadratus lumborum blocks on postoperative pain in arthroscopic hip surgery: a cohort analysis. J Anesth. 2019;33:516-22.

10. Wikner M. Unexpected motor weakness following quadratus lumborum block for gynaecological laparoscopy. Anaesthesia. 2017;72:230-2.

11. Balocco AL, López AM, Kesteloot C, Horn JL, Brichant JF, Vandepitte C, Hadzic A, Gautier P. Quadratus lumborum block: an imaging study of three approaches. Reg Anesth Pain Med. 2021;46:35-40.

12. Kumar A, Sadeghi N, Wahal C, Gadsden J, Grant SA. Quadratus lumborum spares paravertebral space in fresh cadaver injection. Anesth Analg. 2017;125:708-9.

13. Elsharkawy H, Ahuja S, DeGrande S, Maheshwari K, Chan V. Subcostal approach to anterior quadratus lumborum block for pain control following open urological procedures. J Anesth. 2019;33:148-54.

14. Shi R, Li H, Wang Y. Dermatomal coverage of single-injection ultrasound-guided parasagittal approach to anterior quadratus lumborum block at the lateral supra-arcuate ligament. J Anesth. 2021;35:307-10.

15. Hong B, Bang S, Oh C, Park E, Park S. Comparison of PECS II and erector spinae plane block for postoperative analgesia following modified radical mastectomy: bayesian network meta-analysis using a control group. J Anesth. 2021;53:723-33. 
16. Ma J, Bi Y, Zhang Y, Zhu Y, Wu Y, Ye Y, Wang J, Zhang T, Liu B. Erector spinae plane block for postoperative analgesia in spine surgery: a systematic review and meta-analysis. Eur Spine J. 2021. https://doi.org/10.1007/s00586-021-06853-w.

17. Schwartzmann A, Peng P, Maciel MA, Alcarraz P, Gonzalez X, Forero M. A magnetic resonance imaging study of local anesthetic spread in patients receiving an erector spinae plane block. Can J Anesth. 2020;67:942-8.

18. Ma D, Wang R, Wen H, Li H, Jiang J. Cervical erector spinae plane block as a perioperative analgesia method for shoulder arthroscopy: a case series. J Anesth. 2021;35:446-50.
19. Shibata Y, Kampitak W, Tansatit T. The novel costotransverse foramen block technique: distribution characteristics of injectate compared with erector spinae plane block. Pain Phys. 2020;23:E305-14.

Publisher's Note Springer Nature remains neutral with regard to jurisdictional claims in published maps and institutional affiliations. 